\section{Regional anesthesia and pre-existing neurological disease - II}

To the Editor:

Dhir et al. ${ }^{1}$ should be congratulated for successfully administering regional anesthesia to a small series of patients with Charcot-Marie-Tooth disease. In reading the aforementioned case reports, it not only becomes apparent that dissimilar approaches to the brachial plexus were utilized but also that different local anesthetic solutions and volumes were employed. ${ }^{1}$ Universally absent from these local anesthetic solutions was epinephrine. ${ }^{1}$ Epinephrine possesses a complex pharmacodynamic profile and exerts dose dependent changes in peripheral nerve blood flow. ${ }^{2}$ For example, a $1: 200,000$ concentration of epinephrine has been demonstrated to decrease peripheral nerve blood flow. ${ }^{2}$ Unsurprisingly, this reduction in regional blood flow is well tolerated in patients without vascular compromise or peripheral nervous system disorders. ${ }^{2} \mathrm{How}^{-}$ ever, patients afflicted with peripheral nervous system disorders, including Charcot-Marie-Tooth disease, may be more susceptible to nerve damage following periods of diminished blood supply. Maintaining blood flow to a partially demyelinated nerve may eliminate one etiology involved in the "double-crush phenomenon". ${ }^{3}$ Remote from these vascular properties, epinephrine may also enhance local anesthetic induced nerve toxicity. ${ }^{2}$ Given the possible injurious consequences of epinephrine, the authors ${ }^{1}$ should be applauded for omitting this vasoconstrictor from their local anesthetic solutions.

Given the equivocal patient responses to nerve stimulation, ultrasound guidance allowed these practitioners to strategically place local anesthetic circumferentially around the brachial plexus. ${ }^{1}$ The authors do not mention in their article whether the epineurium was violated or if the local anesthetic was placed outside the epineural barrier and allowed to diffuse inward. ${ }^{1}$ When placed underneath the epineurium, local anesthetics may diminish peripheral nerve blood supply secondary to edema or a mass effect. ${ }^{4}$ By not breeching the epineurim and allowing the local anesthetic solution to diffuse inward, it is possible that nerve fascicles in the endoneurium are exposed to a lower concentration of local anesthetic. In general, higher concentrations of local anesthetics produce increased cytotoxic effects. ${ }^{5}$ These cytotoxic effects are typically proportionate to duration of exposure to local anesthetic. ${ }^{5}$ It is worth mentioning that Dhir et al. ${ }^{1}$ did not incorporate bupivacaine into any of their local anesthetic solutions.
Also, the authors did not chronicle tourniquet use in their case reports. ${ }^{1}$ To further eliminate etiologies implicated in the "double-crush phenomenon", ${ }^{3} \mathrm{I}$ believe it is a reasonable request to ask our orthopedic colleagues to forgo tourniquet inflation in patients with known peripheral nervous system disease.

Todd Nelson MD

Colorado Springs, USA

E-mail: nelsontmd@gmail.com

Accepted for publication September 29, 2008.

\section{References}

1 Dbir S, Balasubramanian S, Ross D. Ultrasoundguided peripheral regional blockade in patients with Charcot-Marie-Tooth disease: a review of three cases. Can J Anesth 2008; 55: 515-20.

2 Neal JM. Effects of epinephrine in local anesthetics on the central and peripheral nervous systems: neurotoxicity and neural blood flow. Reg Anesth Pain Med 2003; 28: 124-34.

3 Guay J. First, do no harm: balancing the risks and benefits of regional anesthesia in patients with underlying neurological disease (Editorial). Can J Anesth 2008; 55: 489-94.

4 Neal JM, Bernards CM, Hadzic A, et al. ASRA Practice advisory on neurologic complications in regional anesthesia and pain medicine. Reg Anesth Pain Med 2008; 33: 404-15.

5 Hogan $Q H$. Pathophysiology of peripheral nerve injury during regional anesthesia. Reg Anesth Pain Med 2008; 33: 435-41.

\section{The Authors reply:}

We sincerely thank Drs. Macfarlane and Brull for their interest in our article, and we share their concerns regarding the inability, at the present time, to quantify the 'risks of regional anesthesia $(R A)$.

Patients with Charcot-Marie-Tooth disease (CMT) have had uneventful outcomes under regional anesthesi ${ }^{1,2}$ and adverse outcomes after general anesthesia. ${ }^{3}$ Which technique is right and which is wrong? As Dr. Guay mentioned in the accompanying editorial, ${ }^{+}$we currently do not know the answer to this question. In fact, we are not recommending the use of either regional or general anesthesia in CMT. Our purpose was to inform anesthesiologists that ultrasound guidance might make peripheral nerve block possible in the absence of pre- 
dictable motor response. Our report also emphasizes the importance of discussing, on a case-by-case basis, the risks and benefits of the techniques with the patient prior to surgery.

We agree with Drs. Macfarlane and Brull that a prospective, contemporary, multicentre, national database of $R A$ should be formed. As a first step, we propose a provincial (if not national) SOS regional anesthesia hotline service comparable to that realized by Auroy et al. ${ }^{5}$

We also thank Dr. Nelson for his comments on our article, and for expanding on the role of epinephrine on neural blood flow. In our practice, we use epinephrine at a concentration of 1:400,000. Epinephrine, at this concentration, does not appear to affect nerve blood flow, ${ }^{6}$ and the drug is less likely to cause injury in nerve bundles with intact barrier mechanisms. The barrier mechanism of nerves in patients with CMT disease is unknown, therefore we chose to avoid epinephrine. Commonly a vailable ultrasound technology does not allow one to visually differentiate epineurium from perineurium, however, use of high-resolution ultrasound to differentiate epineurium from perineurium is being investigated.' With ultrasound, it seems feasible to detect as an injectate volume $<1 \mathrm{~mL}$, and thus, to avoid potentially injurious high-volume intraneural local anesthetic injection. ${ }^{8}$ In our report, we were unable to detect epineurium violation and local anesthetic was deposited, in what appeared to be, outside of the epineural barrier.

Shalini Dhir MD

Shyam Balasubramanian FRCA

St. Joseph's Health Care, University of Western Ontario, London, Canada

E-mail: shalini.dhir@sjhc.london.on.ca

\section{References}

1 Dhir S, Balasubramanian S, Ross D. Ultrasound guided peripheral regional blockade in patients with CharcotMarie-Tooth disease: a review of three cases. Can J Anesth 2008: 55: 515-20.

2 Schmitt HJ, Muenster T, Schmidt J. Central neural blockade in Charcot-Marie-Tooth disease. Can J Anesth 2004; 51: 1049-50.

3 Brian JE Jr, Boyles GD, Quirk JG Jr, Clark RB. Anesthetic management for cesarean section of a patient with Charcot-Marie-Tooth disease. Anesthesiology 1987; 66: 410-2.

4 Guay J. First, do no harm: balancing the risks and benefits of regional anesthesia in patients with underlying neurological disease (Editorial). Can J Anesth 2008; 55: 489-94.

5 Auroy $\Upsilon$, Benbamou D, Bargues L, et al. Major complications of regional anesthesia in France:
The SOS Regional Anesthesia Hotline Service.

Anesthesiology 2002; 97: 1274-80.

6 Neal JM. Effects of epinephrine in local anesthetics on the central and peripheral nervous systems: neurotoxicity and neural blood flow. Reg Anesth Pain Med 2003; 28: 124-34.

7 Kubiena H, Hormann M, Michlits W, Tschabitscher $M$, Groszschmidt $K$, Frey $M$. Intraoperative imaging of the brachial plexus by high-resolution ultrasound. J Reconstr Microsurg 2005; 21 : 429-33.

8 Brull R, Chan VW, McCartney CJ, Perlas A, Xu $D$. Ultrasound detects intraneuronal injection. Anesthesiology 2007; 106: 1244.

\section{The Editorialist replies:}

Drs. MacFarlane and Brull propose an audit on the complications of peripheral nerve blocks (PNB) in order to determine whether or not ultrasound guidance (US) can reduce the rate of permanent neurological injury associated with PNBs. Although such an audit would provide valuable information regarding the incidence of this complication in large series of patients, it would be less likely to shed light on the safety of US compared to other methods of nerve localization. For this purpose, a very large randomized controlled trial (RCT) would be required. Since $P N B$ s are associated with a low frequency of major complications, the sample size requirement of such a study would be considerable. Auroy et al. ${ }^{l}$ reported 12 peripheral neuropathies amongst 43,946 PNBs, of which seven were unresolved at six months. This suggests that the incidence of permanent neurological injury associated with PNBs is approximately one per 6,278 interventions. To eliminate a $25 \%$ difference in the incidence of permanent neurological injury associated with PNBs through ultrasonography, this RCT could require a sample size > 2 million subjects to achieve $80 \%$ statistical power.

Is it likely that US will ultimately be found to reduce the incidence of permanent neurological injury associated with PNBs? This seems uncertain. Ultrasound does not prevent intraneural (and bence, possible intrafascicular) injection. ${ }^{2}$ Monitoring the injection pressure and/or the impedance might be other interesting alternatives. ${ }^{3,4}$ For PNBs located proximal to the spine (interscalene, psoas and paravertebral blocks), avoiding orientation of the needle and/or inserting the catheter towards the spine, or at least avoiding needle angle orientations susceptible to facilitate entry into a foramen, and avoiding flexible needles might also help to decrease the risk of severe neurological injury. ${ }^{5,6}$ 
In humans, the addition of epinephrine has not emerged as a risk factor for neurological injury associated with PNB, and bupivacaine-associated neurological injury has been reported inconsistently. To date, no nerve localization or monitoring technique has been shown to be superior in terms of reducing the frequency of clinical injury.' Although the experience with the "perivascular ultrasound-guided technique" is actually less extensipe, there is no reason to think that it would be more prone to induce neurological injury than would be a transaxillary artery technique. A tourniquet may cause neurological damage. High inflation pressures (> 400 $m m \mathrm{Hg}$ ) and longer tourniquet times increase the likelihood of a tourniquet-induced neurological injury. There is no convincing evidence that patients with underlying neurological disease bave an increased susceptibility to any of these factors. In their recently published recommendations, the American Society of Regional Anesthesia suggests the adoption of a prudent approach for these patients: consider avoiding the more potent local anesthetics, reducing the local anesthetic dose and/or concentration, and avoiding or limiting vasoconstrictive additives (epinephrine 1:400,000 or less). ${ }^{7}$ In animal studies, at a concentration of $2.5 \mu g \cdot m L^{-1}$, epinephrine alone produces a transient increase in peripheral nerve blood flow, while at higher concentrations a decrease is most often reported.

Joanne Guay MD FRCPC

Maisonneuve-Rosemont Hospital, University of Montreal, Montreal, Canada.

E-mail: joanne.guay@umontreal.ca

\section{References}

1 Auroy $\Upsilon$, Benhamou D, Bargues L, et al. Major complications of regional anesthesia in France: The SOS Regional Anesthesia Hotline Service. Anesthesiology 2002; 97: 1274-80.

2 Guay J. First, do no harm: balancing the risks and benefits of regional anesthesia in patients with underlying neurological disease (Editorial). Can J Anesth 2008; 55: 489-94.

3 Hadzic A, Dilberovic F, Shah S, et al. Combination of intraneural injection and high injection pressure leads to fascicular injury and neurologic deficits in dogs. Reg Anesth Pain Med 2004; 29: 417-23.

4 Tsui BC, Pillay JJ, Chu KT, Dillane D. Electrical impedance to distinguish intraneural from extraneural needle placement in porcine nerves during direct exposure and ultrasound guidance. Anesthesiology 2008; 109: 479-83.

5 Sardesai AM, Patel R, Denny NM, et al. Interscalene brachial plexus block: can the risk of entering the spinal canal be reduced? A study of needle angles in volunteers undergoing magnetic resonance imaging. Anesthesiology 2006; 105: 9-13.

6 Voermans NC, Crul BJ, de Bondt B, Zwarts MJ, wan Engelen $B G$. Permanent loss of cervical spinal cord function associated with the posterior approach. Anesth Analg 2006; 102: 330-1.

7 Neal JM, Bernards CM, Hadzic A, et al. ASRA Practice advisory on neurologic complications in regional anesthesia and pain medicine. Reg Anesth Pain Med 2008; 33: 404-15.

\section{Gabapentin and post-thoracotomy shoulder pain}

To the Editor:

In their editorial, Drs. MacDougall and Slinger ${ }^{1}$ comment about the wide range of a single dose of preemptive gabapentin (neurontin) studied in clinical trials, i.e., from $300 \mathrm{mg}$ to $1200 \mathrm{mg} .^{2-3}$ This, I believe, underscores a lack of understanding of the pharmacology of gabapentin in the anesthesiology community.

The absorption of gabapentin is dose dependent, secondary to a saturable transport mechanism in the intestine. Hence, the bioavailability of doubling a single dose of gabapentin from 300 to $600 \mathrm{mg}$ decreases from 60 to $40 \%{ }^{4}$ In other words, doubling the dose of gabapentin in the clinical range of 300 to $600 \mathrm{mg}$ will not double the amount of drug absorbed. Instead, it will only increase the quantity of drug absorbed by $33 \%$. This has been noted in a recent systematic review of perioperative gabapentin. ${ }^{5}$ In their paper, Tiippana and colleagues observed that metaregression analysis suggested that the single dose of preemptive gabapentin induced reduction in the 24 -hr opioid consumption was not significantly dependent on the gabapentin dose. The dose studied varied from 300 to $1200 \mathrm{mg}$.

A word of caution: Pfizer Inc. has launched a new drug with properties very similar to gabapentin, i.e., pregabalin (lyrica). In the near future, this drug will likely be studied extensively in the perioperative setting. Although acting on the same receptors, both drugs differ markedly in their absorption characteristics. Contrary to gabapentin, pregabalin has a bioavailability of $90 \%$ that does not vary in the normal range of 25 to $300 \mathrm{mg} \mathrm{po}$ (equivalent to $150 \mathrm{mg}$ to $1800 \mathrm{mg}$ of gabapentin). So, in contrast to gabapentin, increasing doses of pregabalin should significantly increase the bioavailability of the drug, which, in turn, may increase its effectiveness or improve its side-effect profile. 